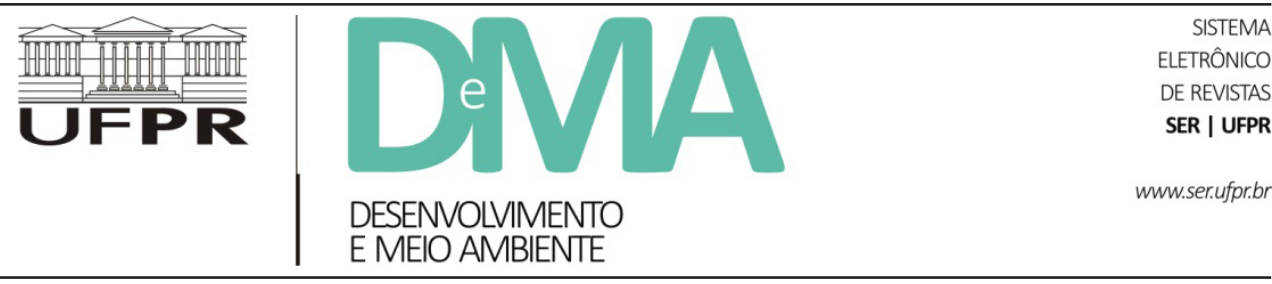

\title{
Produção de mel nos biomas Amazônia, Cerrado e Pantanal, em Mato Grosso, no período de 2002 a 2011
}

\section{Production of Honey in the Biomes of the Amazon, Cerrado (Bushlands) and Pantanal (Swamplands) of Mato Grosso between 2002 and 2011}

\author{
Jennifer Oberger FERREIRA ${ }^{1}$, Carla GALBIATI ${ }^{2 *}$, Sandra Mara Alves da Silva NEVES ${ }^{3}$, Mirian Raquel da \\ Silva MIRANDA ${ }^{4}$ \\ ${ }^{1}$ Programa de Pós-Graduação em Ciências Ambientais, Universidade do Estado de Mato Grosso (Unemat), Sinop, MT, Brasil. \\ ${ }^{2}$ Departamento de Agronomia, Universidade do Estado de Mato Grosso (Unemat), Sinop, MT, Brasil. \\ ${ }^{3}$ Departamento de Geografia, Universidade do Estado de Mato Grosso (Unemat), Sinop, MT, Brasil. \\ ${ }^{4}$ Programa de Pós-Graduação em Ambiente e Sistemas de Produção Agrícola, Universidade do Estado de Mato Grosso (Unemat), Tangará da \\ Serra, MT, Brasil. \\ *E-mail de contato: carla@unemat.br
}

Artigo recebido em 26 de abril de 2016, versão final aceita em 10 de novembro de 2016.

RESUMO: A apicultura é uma atividade econômica que utiliza o ambiente natural, sem desflorestar, e promove o serviço de polinização de espécies cultivadas em ambiente antrópico. Objetivou-se analisar a produção de mel nos biomas mato-grossenses Amazônia, Cerrado e Pantanal, no período 2002 a 2011. As informações de produção de mel por município foram obtidas em bancos de dados do Instituto Brasileiro de Geografia e Estatística (IBGE). A delimitação dos municípios por bioma foi definida de acordo com o IBGE. A produção de mel foi maior na Amazônia, com 46,88\% da produção estadual, e houve a participação de 58 municípios desse bioma; o Cerrado produziu $42 \%$ do mel do Estado, com a participação de 50 municípios produtores; e o Pantanal, por sua vez, produziu $10,82 \%$ do mel, com oito municípios. Conclui-se que os biomas mato-grossenses apresentaram potencial apícola, com maior capacidade produtiva no Pantanal, devido à maior produtividade.

Palavras-chave: apicultura; uso e ocupação do solo; sensoriamento remoto; conservação ambiental.

ABSTRACT: Beekeeping is an economic activity that uses the natural environment without forest degradation and promotes the service of pollination of the species cultivated in an anthropic environment. The purpose of this study was to analyze honey production in Mato Grosso biomes of the Amazon, Cerrado (Bushlands) and Pantanal (Swamplands) from 2002 to 2011. Information on the production of honey by each municipality was obtained from the Brazilian Institute of Geography and Statistics database (IBGE). The delimitation of the municipalities for each biome was defined according to IBGE. The production of honey was the greatest in the Amazon, with $46.88 \%$ of the State production and the participation of 58 municipalities from this biome; 
Cerrado (Bushlands) produced $42 \%$ of the State's honey with the participation of 50 producing municipalities, and Pantanal (Swamplands) produced $10.82 \%$ of the honey with 8 municipalities. We conclude that the Mato Grosso biomes showed beekeeping potential, with greater productive capacity in Pantanal (Swamplands) due to its higher productivity rate.

Keywords: beekeeping; land-use and occupation; remote sensing; environmental conservation.

\section{Introdução}

As culturas agrícolas e pastagem são sistemas produtivos influenciados pelas vegetações naturais em que estão inseridas. Isso foi observado na Itália, em Portugal, na Espanha e no Brasil, em floresta e savana (Carreiras et al., 2006; Kloverpris, 2009). Os sistemas produtivos no Brasil têm recebido influência positiva de fatores como as condições do solo e do clima nos estados do Paraná, da Paraíba e de Goiás (Caires et al., 2004; Silva et al., 2009).

Em Mato Grosso, 36,9\% do território está ocupado pela agropecuária (Carreiras et al., 2006), que constitui o principal sistema produtivo, devido ao ambiente favorável proporcionado pela vegetação, clima, solo e relevo do estado, segundo o Instituto Mato-Grossense de Economia Agropecuária (IMEA, 2013). A ampliação das áreas de agricultura e pastagem, aliada à expansão dos centros urbanos e ao crescimento da industrialização, tem causado a deterioração dos recursos naturais, principalmente do solo e da água (Campos et al., 2004; Silva et al., 2009).

Para conter a pressão antrópica sobre o ambiente, o governo de Mato Grosso desenvolveu, com o auxílio da sociedade, um plano de desenvolvimento que objetiva, em 20 anos (2006-2026), executar projetos e ações que explorem as potencialidades do estado, assegurando o desenvolvimento sustentável (Mato Grosso, 2012).

A apicultura atende aos objetivos desse plano de desenvolvimento de forma direta, pois amplia a geração de emprego e renda da população, conserva o meio ambiente e a biodiversidade, reduz o desmatamento, diversifica a estrutura produtiva e melhora a qualidade de vida do apicultor e do consumidor de produtos apícolas (Santos \& Ribeiro, 2006; Fujji et al., 2009; Mato Grosso, 2012).

A flora é o fator preponderante para obter-se um bom resultado na apicultura, como apontado pelos pesquisadores Sodré et al. (2008). A riqueza de espécies, a quantidade de flores e a proximidade de áreas nativas de floresta influenciam positivamente a produção e a qualidade do mel (Sande et al., 2009; Alves et al., 2011; Amaral et al., 2012). No Cerrado e no Pantanal, a apicultura tem se destacado na produção de mel devido, principalmente, à riqueza de espécies e à quantidade de plantas com alto potencial apícola existente nesses biomas (Pott \& Pott, 1986; Sodré et al., 2008; Sande et al., 2009; Alves et al., 2011).

Apesar do potencial apícola existente em Mato Grosso, o estado ainda ocupa o $16^{\circ}$ lugar na produção de mel do país, com uma produção de 379 toneladas (IBGE, 2013). Assim sendo, a implantação de política econômica de apoio à apicultura pode favorecer o desenvolvimento da atividade, porém estudos de viabilidade dos locais mais promissores para a atividade são importantes na orientação de ações e investimentos. Mato Grosso possui um território extenso, em que 53,6\% são ocupados pelo bioma Amazônico, 39,6\% pelo Cerrado e 6,79\% pelo Pantanal (Mato Grosso, 2013a). Esses biomas são ricos em espécies vegetais que podem contribuir de forma direta na apicultura, devido à extensão que ocupam no Estado e a sua diversidade intrínseca. 
Os mapas na atualidade, gerados por meio das geotecnologias (Sistema de Informação Geográfica, sensoriamento remoto, GPS etc.), constituem instrumentos indispensáveis para o planejamento, gestão e tomada de decisão quanto à definição do uso da terra e da cobertura vegetal para diferentes unidades territoriais (países, estados, regiões, municípios etc.). A utilização de imagens está se tornando cada vez mais comum, devido à potencialidade dada ao acompanhamento e à avaliação da dinâmica de ocupação do uso da terra (Souza et al., 2012). Esse recurso cartográfico pode ser utilizado na caracterização da distribuição geográfica do mel, contribuindo assim para o fortalecimento da atividade apícola. O uso da geotecnologia no estudo da apicultura foi realizado por Amaral et al. (2012), Loureiro et al. (2013) e Marochi et al. (2013).

Nesse contexto, o objetivo desta pesquisa foi analisar a produção de mel nos biomas mato-grossenses Amazônia, Cerrado e Pantanal, no período de 2002 a 2011.

\section{Material e métodos}

\subsection{Local de estudo}

O estado de Mato Grosso possui uma extensão territorial de 903.366,192 km² (IBGE, 2013), em que ocorrem três distintos biomas brasileiros: Amazônia, Cerrado e Pantanal. O bioma Amazônia no território de estudo caracteriza-se por diferentes aspectos fitofisionômicos, visto que a diversidade em área de preservação ambiental na Amazônia mato-grossense foi de 1.366 espécies botânicas, e estima-se que esse bioma abrigue cerca de $40 \mathrm{mil}$ espécies vasculares de plantas (Mittermeier et al., 2003; Zappi et al., 2011). No Cerrado mato-grossense foram encontradas 80 espécies botânicas em uma área de $1.800 \mathrm{~km}^{2}$ (Felfili et al., 2002), revelando a elevada biodiversidade desse bioma. O Pantanal, na área de estudo, possui florestas sempre verdes, sazonalmente inundadas, e permanecem secas, durante o período de estiagem (junho a novembro), florestas livres de inundação, campos limpos com baixa densidade de árvores e habitats aquáticos, com cerca de 2 mil espécies de plantas catalogadas (Fernandes et al., 2010; Mato Grosso, 2013b).

Em Mato Grosso, o bioma Amazônia está presente em 67 municípios; o Cerrado, em 65; e o Pantanal, em 9 (Figura 1).

A população mato-grossense corresponde a 3.035.122 habitantes, em que $81,8 \%$ vivem na área urbana e 18,2\% na área rural (IBGE, 2013). As principais atividades econômicas são a pecuária e a agricultura (Mato Grosso, 2012). Existem 21.545 famílias de agricultores familiares, entre elas assentados e pequenos agricultores (MDA, 2013), e, desses, 1.135 são apicultores, organizados em 30 associações, uma cooperativa, uma federação, seis casas de mel e três entrepostos registrados em Mato Grosso (Dallemole et al., 2010).

\subsection{Obtenção dos dados}

Para analisar a produção de mel realizada nos municípios contidos nos biomas de Mato Grosso, foi selecionada uma década, correspondente ao período de 2002 a 2011. As informações sobre a produção e os municípios mato-grossenses foram obtidas em bancos de dados secundários on-line do Instituto Brasileiro de Geografia e Estatística (IBGE, 2013).

A produção de mel foi quantificada por meio da somatória da produção anual em cada município mato-grossense na década de estudo, enquanto a por bioma foi quantificada pela somatória dos municípios nele situados. A quantidade de mel produzido 


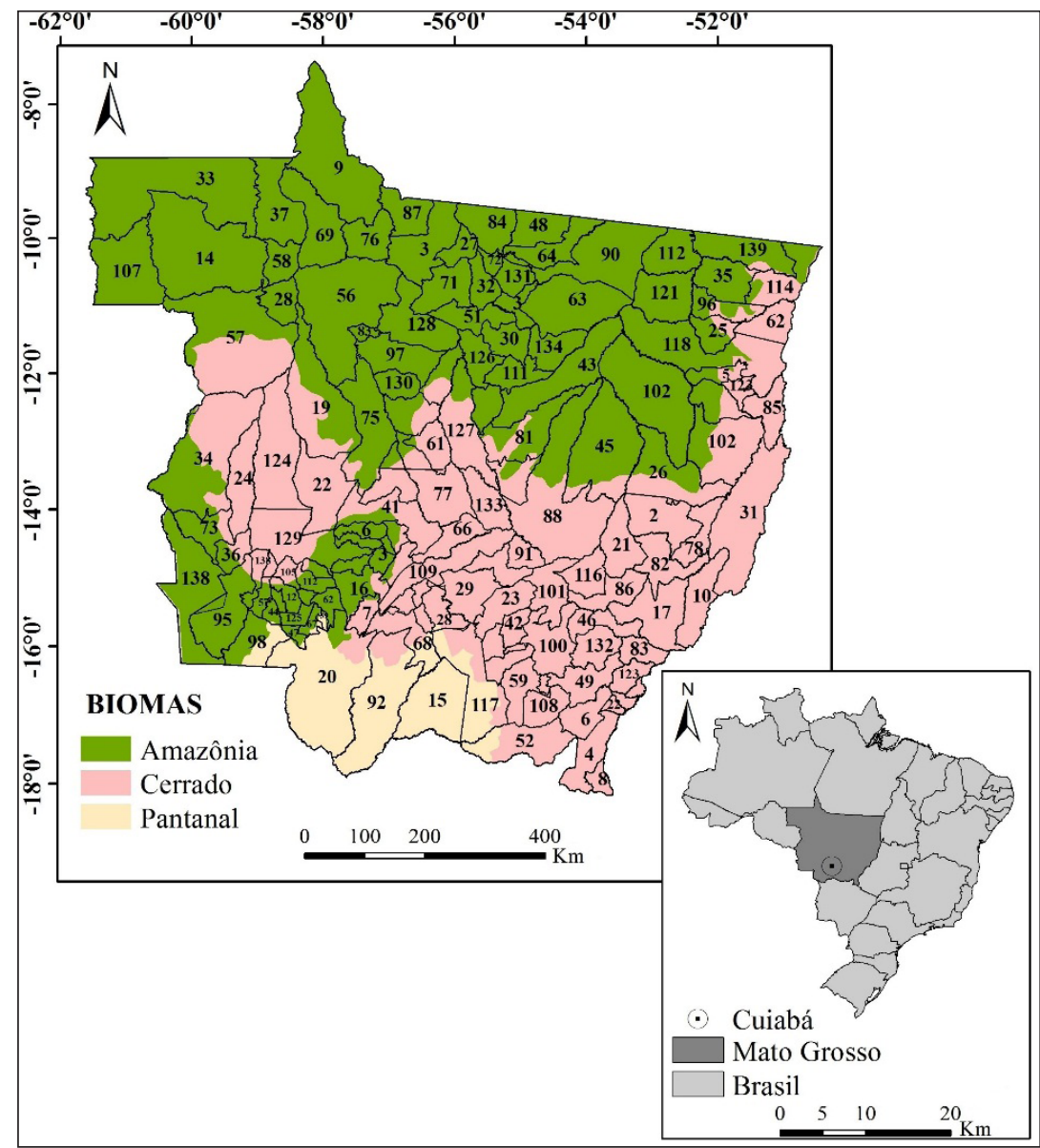

FIGURA 1 - Localização dos municípios de Mato Grosso nos biomas Amazônia, Cerrado e Pantanal.

Elaboração: Jennifer Oberger Ferreira, 2014

por bioma foi mensurada pela razão entre o somatório da produção de mel do município na década (2002 a 2011) e o somatório da produção de mel dos municípios do bioma na década em porcentagem (Fórmula 1). A proporção de produção de mel no município em relação ao estado foi quantificada usando-se a mesma equação, exceto o que foi usado, no denominador da fórmula, o somatório da produção de mel dos municípios do estado. A produção de mel/área do bioma foi calculada a partir da razão entre a somatória da produção e a área dos biomas.

$$
P_{m}=\frac{\sum_{i=1}^{0} P_{i}}{\sum_{i=1}^{0}\left(\sum_{j=1}^{1} P_{j}\right)} * 100
$$

onde:

$P_{m}=$ produção de mel;

$P_{i}=$ produção de mel do município na década;

$i=1, \ldots, 10$ (quantidade de anos analisados); bioma). 
A produção total de mel por município, no período de dez anos (2002 a 2011), foi agrupada pela frequência devido à amplitude total ser de zero a $201.500 \mathrm{~kg}$ de mel. Para isso usou-se divisão por quartis, valores que dividem a série em três partes iguais, com 33\% das observações em cada quartil de frequência.

Nos municípios que apresentaram mais de um bioma em seu limite territorial foi considerado para análise aquele que possuía maior extensão no município.

Os mapas da evolução da produção de mel no período de 2002 a 2011 foram elaborados para as classes de produção dos biomas e dos municípios. Na elaboração dos mapas da evolução da produção dos biomas e municípios do estado de Mato Grosso, foram utilizados arquivos vetoriais, na extensão shapefile (shp), dos sites do Ministério do Meio Ambiente e do IBGE. A inserção das informações das classes de produção na tabela de dados do arquivo vetorial dos municípios foi realizada no Sistema de Informação Geográfica (SIG).

\section{Resultados e discussão}

\subsection{Produção de mel no estado}

No bioma Amazônia, 80\% dos municípios, que representam 58 deles, produziram mel na década estudada, totalizando $1.602 .481 \mathrm{~kg}$, o que corresponde a $46,88 \%$ da produção total de mel no Estado. A produção de mel/área foi de $3,30 \mathrm{~kg} / \mathrm{km}^{2}$. Nesse bioma, 13 municípios produziram até 1.200 $\mathrm{kg}$ de mel; 37, até $30.012 \mathrm{~kg}$; e 17 municípios produziram acima de $30.012 \mathrm{~kg}$ (Tabela 1). Esses valores demonstram que 17 municípios se destacaram na produção, pois foram responsáveis por $74,10 \%$ da produção total de mel do bioma e $34 \%$ da produção do Estado. Os outros 50 municípios desse bioma produziram $25,9 \%$ do total da produção de mel. O fato de o bioma Amazônia ser o maior produtor de mel no Estado pode estar ligado à sua abrangência territorial, que ocupa uma extensão de 53,6\% do estado, a maior dentre os três biomas. Isso foi confirmado ao se analisar a produção de mel/área do bioma, que foi a menor entre os biomas analisados.

O Cerrado possuía 50 municípios produtores de mel na década investigada, correspondendo a $85 \%$ dos municípios do bioma e contribuindo com $42,25 \%$ da produção total de mel do Estado. A produção de mel/área desse bioma foi de 4,03 $\mathrm{kg} / \mathrm{km}^{2}$. Assim como na Amazônia, a maioria dos municípios (43\%) produz entre $1.201 \mathrm{~kg}$ e 30.012 $\mathrm{kg}$ de mel, e 16 municípios, ou seja, 24\% dos municípios produtores do Cerrado foram responsáveis por $80 \%$ da produção total de mel do bioma e $34 \%$ da produção do Estado (Tabela 1). Os outros 20\% da produção de mel foram produzidos pelos 49 municípios, indicando que no Cerrado $1 / 4$ dos municípios apresenta maior aptidão para a produção de mel do que os demais municípios.

Dos nove municípios do bioma Pantanal, oito contribuíram com $10,87 \%$ da produção de mel do Estado na década. A produção de mel/área desse bioma foi de $6,05 \mathrm{~kg} / \mathrm{km}^{2}$. Dos oito municípios produtores, Cáceres e Poconé contribuíram com $76,1 \%$ da produção total de mel do bioma e com $8,27 \%$ do Estado. A maioria dos municípios produziu até $30.012 \mathrm{~kg}$ de mel (Tabela 1). No Pantanal, a média da produção de mel dos municípios foi de $41.278,89 \pm 7.322 \mathrm{~kg}$, superior à média dos outros biomas (Amazônia $=23.917,63 \pm 5.514$ e Cerrado $=22.221,57 \pm 4.770)$. Além disso, a produção/área do bioma foi maior no Pantanal, confirmando essa superioridade. Esse resultado pode estar relacionado à organização da atividade apícola no Pantanal, que possui associações de apicultores e conta com 
a parceria do Sebrae (Dotto et al., 2008; Dallemole et al., 2010).

A produção/área de mel no Pantanal foi maior que nos outros biomas em Mato Grosso devido à contribuição das espécies presentes nas áreas de pastagens para pecuária, como gramíneas e algumas espécies arbóreas. As pastagens associadas a vegetação de savana foram frequentes em apiários de Mato Grosso (Amaral et al., 2012), com oferta farta de área de pastoreio. As espécies arbóreas Curatella americana (Dilleniaceae) (lixeira) e Astronium fraxinifolium (Anacardiaceae) (aroeira-do-campo) estão presentes em Mato Grosso em domínios fitogeográficos de Cerrado (Fraga \& Paula-Souza, 2015; Silva-Luz \& Pirani, 2015). Essas duas espécies foram consideradas importantes no pastoreio das abelhas (Da Silva et al., 2000); $A$. fraxinifolium foi comum no mel do Pantanal, e $C$. americana, na vegetação (Silva \& Galbiati, 2010). Em vegetação tipo savana e floresta, as espécies arbóreas têm contribuído menos com a produção de mel (Da Luz et al., 2007), excetos pelas cultivadas como Eucalyptus sp e Citrus sp (Mendonça et al., 2008; Simeão et al., 2015).

Além disso, o bioma Pantanal possui $77 \%$ do seu território conservado a maior área de preservação em relação aos outros dois biomas estudados, enquanto a Amazônia possui 65\%, e o Cerrado, 53\% do território preservado, o que pode ter contribuído com o resultado encontrado (Amaral et al., 2012; Dallemole et al., 2010; Mato Grosso, 2013b).

TABELA 1 - Três classes de produção de mel (kg) em Mato Grosso e nos biomas, no período de 2002 a 2011, em relação ao número de municípios produtores de mel, à proporção de produção nos biomas e à proporção no estado

\begin{tabular}{|c|c|c|c|c|c|}
\hline $\begin{array}{c}\text { Biomas/Classes de } \\
\text { produção de mel (kg) }\end{array}$ & $\begin{array}{l}\mathrm{N}^{0} \text { de muni- } \\
\text { cípios }\end{array}$ & $\begin{array}{l}\text { Produção de } \\
\text { mel (kg) }\end{array}$ & $\begin{array}{c}\text { Produção no } \\
\text { bioma (\%) }\end{array}$ & $\begin{array}{c}\text { Produção de MT } \\
(\%)\end{array}$ & $\begin{array}{c}\text { Produção/Área do } \\
\text { bioma* }\end{array}$ \\
\hline \multicolumn{6}{|l|}{ Amazônia } \\
\hline Classe $1(0-1.200)$ & 13 & 2.266 & 0,14 & 0,06 & 0,005 \\
\hline Classe $2(1.201-30.012)$ & 37 & 412.748 & 25,76 & 12,08 & 0,85 \\
\hline $\begin{array}{l}\text { Classe } 3 \text { (30.013 - } \\
\text { 201.452) }\end{array}$ & 17 & 1.187 .467 & 74,10 & 34,74 & 2,45 \\
\hline Subtotal & 67 & 1.602 .481 & 100 & 46,88 & 3,30 \\
\hline \multicolumn{6}{|l|}{ Cerrado } \\
\hline Classe $1(0-1.200)$ & 21 & 3.369 & 0,24 & 0,10 & 0,01 \\
\hline Classe $2(1.201-30.012)$ & 28 & 278.521 & 19,28 & 8,15 & 0,78 \\
\hline $\begin{array}{l}\text { Classe } 3 \text { (30.013 - } \\
\text { 201.452) }\end{array}$ & 16 & 1.162 .512 & 80,48 & 34,00 & 3,24 \\
\hline Subtotal & 65 & 1.444 .402 & 100 & 42,25 & 4,03 \\
\hline \multicolumn{6}{|l|}{ Pantanal } \\
\hline Classe $1(0-1.200)$ & 1 & 0 & 0,00 & 0,00 & 0,00 \\
\hline Classe $2(1.201-30.012)$ & 6 & 88.809 & 23,9 & 2,60 & 1,45 \\
\hline $\begin{array}{l}\text { Classe } 3(30.013- \\
201.452)\end{array}$ & 2 & 282.701 & 76,1 & 8,27 & 4,60 \\
\hline Subtotal & 9 & 371.510 & 100 & 10,87 & 6,05 \\
\hline Mato Grosso & 141 & 3.418 .393 & - & 100 & \\
\hline
\end{tabular}

* Produção de mel/área do bioma $\left(\mathrm{kg} / \mathrm{km}^{2}\right)$ 
3.2. Evolução da produção de mel de 2002 a 2011 no estado

No ano de 2002, dos 67 municípios que estão situados no bioma Amazônia, 46 municípios produziam mel; dos 65 do Cerrado, 30 municípios eram produtores; e dos nove do Pantanal somente quatro municípios tiveram produção (Figura 2). Em 2011, houve uma redução no número de municípios produtores de mel no bioma Amazônia, de 46 para 42 municípios. No Cerrado, a situação foi inversa: aumentou de 30 para 37 municípios produtores; e, no Pantanal, de quatro para oito municípios produtores. Portanto, a evolução da produção de mel, de 2002 a 2011, evidencia um crescimento no número de municípios produtores no Estado, de 80 para 87.
Na Amazônia, entre 2002 e 2011, começaram a produzir mel oito municípios, que são: Cotriguaçu, São José do Xingu, Cláudia, Colniza, Nortelândia, Nova Santa Helena, Rondolândia e Santa Cruz do Xingu, enquanto, em 2011, 13 não apresentaram produção: Arenápolis, Barra do Bugres, Nova Marilândia, Salto do Céu, Santo Afonso, União do Sul, Alto da Boa Vista, Colíder, Matupá, Nova Canaã do Norte, Nova Monte Verde, Rio Branco e Terra Nova do Norte (Figura 2).

No Cerrado, 13 municípios foram introduzidos na produção de mel no período averiguado, são eles Alcorizal, Cocalinho, Juscimeira, Planalto da Serra, Campos de Júlio, Conquista d'Oeste, Ipiranga do Norte, Itiquira, Nova Brasilândia, Nova Lacerda, Ribeirão Cascalheira, Rosário Oeste e Vale de São



FIGURA 2 - Evolução da produção apícola de Mato Grosso de 2002 e 2011 nos biomas. Os municípios produtores de mel estão identificados com malha reticular. 
Domingos, enquanto seis deixaram de produzir: Alto Paraguai, General Carneiro, Alto Araguaia, Alto Taquari, Santa Terezinha e Sapezal (Figura 2).

No Pantanal, os municípios Cáceres, Cuiabá, Mirassol d'Oeste e Santo Antônio do Leverger foram produtores de mel no período de 2002 a 2011, e, em 2011, mais quatro tornaram-se produtores: Barão de Melgaço, Nossa Senhora do Livramento, Poconé e Várzea Grande (Figura 2).

Em relação à quantidade de mel, a Amazônia produziu $75.450 \mathrm{~kg}$ em 2002, com 30 municípios na classe 2 e 16 municípios na classe 3. Em 2011, o número de municípios produtores diminuiu na classe 2 para 28 e na classe 3 para 14. Mesmo com essa redução, o bioma Amazônia aumentou a produção para $204.828 \mathrm{~kg}$ de mel em 2011 (Tabela 2). Isso pode ser indicativo para o aumento da produtividade (produção de mel/colmeia).

O Cerrado superou a produção da Amazônia em 2002, com $90.887 \mathrm{~kg}$ de mel. Naquele bioma, em 2002, 13 municípios estavam na classe 2 e 17 na classe 3. Em 2011, 18 municípios estavam inseridos na classe 2 e 19 na classe 3 , aumentando a produção do Cerrado para $135.500 \mathrm{~kg}$ de mel (Tabela 2).

Com três municípios na classe 2 e apenas um município na classe 3 , o Pantanal produziu 8.508 $\mathrm{kg}$ de mel em 2002. Em 2011, o número aumentou para seis municípios produtores na classe 2 e dois municípios produtores na classe 3 , totalizando $38.953 \mathrm{~kg}$ de mel produzidos (Tabela 2).
No bioma Amazônia, os municípios de Marcelândia, Nova Bandeirantes, Nova Ubiratã, Pontes e Lacerda, Porto Esperidião e Santa Carmem aumentaram sua produção no período analisado e foram agrupados, em 2011, na classe 3. No entanto, em Carlinda, Feliz Natal, Itaúba, Juara, Novo Mundo, Paranaíta, Sinop e Vila Rica, a produção foi reduzida e esses municípios passaram, assim, a integrar a classe 2 em 2011. Isso demonstra que, além de o número de municípios produtores ter decrescido, no período de 2002 para 2011, no bioma Amazônia, a maioria deles reduziu a produção (Figura 3).

A redução do número de municípios produtores de mel e da quantidade de mel produzido no bioma Amazônia, em 2011, pode estar relacionada à expansão da atividade agropecuária, em especial a pecuária de corte e o cultivo de soja, milho, arroz e algodão, o que ocorreu em grande escala nas terras sob influência desse bioma (IBGE, 2013). Essa expansão implica a realocação das atividades existentes, substituindo-as pelas culturas produzidas em larga escala (Rivero et al., 2009).

No Cerrado, os municípios de Brasnorte, Campo Novo do Parecis, Reserva do Cabaçal e Rondonópolis tiveram aumento na produção de mel entre 2002 e 2011 e foram inseridos na classe 3. Entretanto, Nova Nazaré, Paranatinga, Poxoréu, Canarana e Nova Xavantina apresentaram decréscimo na quantidade de mel, o que resultou na mudança deles da classe 3 para a 2 (Figura 3).

TABELA 2 - Produção de mel nos três biomas de Mato Grosso e número de municípios em duas classes, nos anos de 2002 e 2011

\begin{tabular}{|c|c|c|c|c|c|c|}
\hline \multirow{3}{*}{ Biomas } & \multicolumn{3}{|c|}{2002} & \multicolumn{3}{|c|}{2011} \\
\hline & \multirow{2}{*}{ Produção (kg) } & \multicolumn{2}{|c|}{ Número de municípios } & \multirow{2}{*}{ Produção (kg) } & \multicolumn{2}{|c|}{ Número de municípios } \\
\hline & & Classe 2 & Classe 3 & & Classe 2 & Classe 3 \\
\hline Amazônia & 75.450 & 30 & 16 & 204.828 & 28 & 14 \\
\hline Cerrado & 90.887 & 13 & 17 & 135.500 & 18 & 19 \\
\hline Pantanal & 8.508 & 3 & 1 & 38.953 & 6 & 2 \\
\hline
\end{tabular}






FIGURA 3 - Produção de mel nos biomas de Mato Grosso em 2002 na classe 2 (a) e classe 3 (c) e em 2011 na classe 2 (b) e classe 3 (d). Os municípios produtores de mel estão identificados com malha reticular. 
Apesar de o Cerrado possuir extensas áreas de cultivo de grãos e criação de gado, semelhante à Amazônia, essas áreas estão consolidadas. Enquanto, na Amazônia, há a substituição de atividades de pequeno porte, como a apicultura, para a inserção dos cultivos em larga escala, no Cerrado ocorre o processo inverso, com a introdução da apicultura como atividade secundária em áreas cultivadas, pois são áreas cultivadas há muito tempo (Reis \& Comastri Filho, 2003).

No Pantanal, os municípios de Cuiabá, Várzea Grande, Nossa Senhora do Livramento, Santo Antônio do Leverger, Poconé e Mirassol d'Oeste mantiveram a produção de mel na classe 2 , e os municípios de Cáceres e Barão de Melgaço permaneceram na classe 3 (Figura 3).

Ao contrário do ocorrido no bioma Amazônia, no Cerrado e no Pantanal houve aumento no número de municípios produtores de mel entre 2002 e 2011. Esse crescimento pode estar relacionado com o aumento de preço devido ao início das exportações de mel nesse período (Amaral, 2010). Além disso, o Estado incentivou a produção de mel por meio de projetos de compra do mel dos produtores de alguns municípios desses biomas para distribuição em asilos, escolas e creches (SEBRAE, 2013).

A diversidade floral existente no tipo de vegetação de matas favoreceu a produção de mel por disponibilizar abundante recurso alimentar para as colmeias (Alves et al., 2011). Santos e Ribeiro (2006) constataram que a diversidade de floradas das matas mato-grossenses proporciona um grande potencial para desenvolver a apicultura no estado.

Para aumentar a produção apícola é necessário identificar as áreas de vegetação visitadas pelas abelhas, para indicar quais são as melhores para a instalação das colmeias (Marochi et al., 2013). Além disso, é necessário desenvolver projetos políticos e econômicos que auxiliem o planejamento e a gestão para o desenvolvimento da apicultura e que estruturem as associações e capacitem os apicultores (Dallemole et al., 2010). A análise do uso e da ocupação do solo auxiliou no planejamento e gestão do setor econômico, como o turismo (Lopes \& Ruchkys, 2015), e na preservação e conservação dessa unidade (Muller \& Bessa Junior, 2008), assim como se mostrou promissora para a apicultura em Mato Grosso.

A análise temporal da produção de mel e do uso e ocupação do solo (cobertura vegetal), no estado de Mato Grosso, no período do estudo de dez anos, não foi possível devido ao fato de os dados de cobertura vegetal disponíveis no IBGE serem do ano de 2006.

A taxa de desmatamento nos biomas de Mato Grosso é alta, especialmente na Amazônia e no Cerrado, e a apicultura pode contribuir para reduzir essas taxas quando implantada em áreas preservadas, ao mesmo tempo em que pode ser utilizada como atividade secundária em áreas agrícolas e de pastagem (Reis \& Comastri Filho, 2003; Cardoso et al., 2009). A conservação de áreas de florestas deve ser incentivada por políticas econômicas com maior valor social, como foi verificado por Andrade et al. (2012), em termos de serviços ecossistêmicos em unidades de conservação.

\section{Considerações finais}

Mato Grosso apresenta potencial apícola e a apicultura pode ser implantada em áreas antropizadas e de vegetação nativa nos três biomas presentes no estado.

O Pantanal apresentou o melhor desempenho na produção de mel, com média e produção/área superiores às dos outros biomas. $\mathrm{O}$ bioma Amazônia apresentou menor desempenho da apicultura, com 
a menor produção/área do Estado e na década estudada, redução do número de municípios produtores de mel. No Cerrado, a produção de mel encontra-se em expansão, decorrente do aumento do número de municípios produtores.

\section{Referências}

Alves, E. M.; Sereia, M. J.; Toledo, V. A. A. S.; Marchini, L. C.; Neves, C. A.; Toledo, T. C. S. O. A.; Anacleto, D. A. Physicochemical characteristics of organic honey samples of africanized honeybees from Parana River island. Ciência e Tecnologia de Alimentos, 31, 635-639, 2011. doi: 10.1590/ S0101-20612011000300013

Amaral, A. M. Arranjo produtivo local e apicultura como estratégias para o desenvolvimento do sudoeste de Mato Grosso. São Carlos, Tese (Doutorado em Ecologia e Recursos Naturais) - UFSCAR, 2010.

Amaral, A. M.; Galbiati, C.; Barelli, M. A. A.; Neves, S. M. A. S.; Margarido, L. A. C. Uso da terra e produção de mel na região sudoeste de Mato Grosso, Brasil. In: Santos, J. E., Silva, C. J.; Moschini, L. E. (Orgs.). Paisagem, biodiversidade e cultura. São Carlos: Rima, 2012. p. 121-142.

Andrade, D. C.; Romeiro. A. R.; Fasiaben, M. do C. R.; Garcia, J. R. Dinâmica do uso do solo e valoração de serviços ecossistêmicos: notas de orientação para políticas ambientais. Desenvolvimento e Meio Ambiente, 25, 53-71, 2012. doi: $10.5380 /$ dma.v25i0.26056

Caires, E. F.; Kusman, M. T.; Barth, G.; Garbuio, F. J.; Padilha, J. M. Alterações químicas do solo e resposta do milho à calagem e aplicação de gesso. Revista Brasileira de Ciência do Solo, 28, 125-136, 2004. doi: 10.1590/S010006832004000100013

Campos, S.; Araújo Júnior, A. A.; Barros, Z. X.; Cardoso, L.; Piroli, E. L. Sensoriamento remoto e geoprocessamento aplicados ao uso da terra em microbacias hidrográficas, Botucatu - SP. Engenharia Agrícola, 24, 431-435, 2004. doi: 10.1590/S0100-69162004000200023

Cardoso, E. L.; Silva, M. L. N.; Moreira, F. M. S.; Curi, $\mathrm{N}$. Atributos biológicos indicadores da qualidade do solo em pastagem cultivada e nativa no Pantanal. Pesquisa

\section{Agradecimentos}

À Coordenação de Aperfeiçoamento de Pessoal de Nível Superior (Capes) pela concessão da bolsa de estudo à primeira autora.

Agropecuária Brasileira, 44, 631-637, 2009. doi: 10.1590/ S0100-204X2009000600012

Carreiras, J. M. B.; Pereira, J. M. C.; Campagnolo, J. M. C.; Shimabukuro, Y. E. Assessing the extent of agriculture/ pasture and secondary succession forest in the Brazilian Legal Amazon using SPOT VEGETATION data. Remote Sensing of Environment, 101, 283-298, 2006. doi: 10.1016/j. rse.2005.12.017

Da Luz, C. F. P.; Thomé, M. L.; Bart, O. M. Recursos tróficos de Apis mellifera L. (Hymenoptera, Apidae) na região de Morro Azul do Tinguá, Estado do Rio de Janeiro. Revista Brasileira de Botânica, 30(1), 29-36, 2007. doi: 10.1590/ S0100-84042007000100004

Dallemole, D.; Faria, A. M. M.; Azevedo-Junior, W. C.; Gomes, V. M. O arranjo produtivo local da apicultura de Mato Grosso: evolução recente e necessidade de ajustes. Revista de Estudos Sociais, 2, 181-197, 2010. Disponível em: <http://periodicoscientificos.ufmt.br/index.php/res/ article/view/290/259>.

Dotto, S. E.; Pimentel, C. C. R.; Campos, H. L. S. Redes produtivas: um estudo de caso da Associação Retirense de Apicultores em Barão de Melgaço - MT como alternativa de desenvolvimento regional. Espacio y Desarrollo, 20, 147-159, 2008. Disponível em: <http://revistas.pucp.edu. pe/index.php/espacioydesarrollo/article/view/5457/5454>.

Felfili, J. M.; Nogueira, P. E.; Silva Júnior, M. C.; Marimon, B. S.; Delitti, W. B. C. Composição florística e fitossociologia do cerrado sentido restrito no município de Água Boa - MT. Acta Botânica Brasileira, 16, 103-112, 2002. doi: 10.1590/S0102-33062002000100012

Fernandes, I. M.; Signor, C. A.; Penha, J. Biodiversidade no Pantanal de Poconé. Cuiabá: Attema, 2010. 196 p. 
Fraga, C. N.; Paula-Souza, J. Dilleniaceae in Lista de Espécies da Flora do Brasil. Jardim Botânico do Rio de Janeiro, 2015, Disponível em: <http://floradobrasil.jbrj.gov.br/jabot/ floradobrasil/FB7337>. Acesso em: 20/10/2016.

Fujji, I. A.; Rodrigues, P. R. M.; Ferreira, M. N. Caracterização físico-química do mel de guaranazeiro (Paullinia cupana var. sorbilis) em Alta Floresta, Mato Grosso. Revista Brasileira de Saúde e Produção Animal, 10, 645-653, 2009. Disponível em: $<$ http://www.rbspa.ufba.br/index.php/rbspa/ article/view/1185/855>.

IBGE - Instituto Brasileiro de Geografia e Estatística. Censo Agropecuário 2006, 2006. Disponível em: <http://www. ibge.gov.br>. Acesso em: ago. 2013.

IBGE - Instituto Brasileiro de Geografia e Estatística. $\mathrm{Ci}$ dades, 2013, Disponível em: <http://cidades.ibge.gov.br/ xtras/uf.php? coduf=51>. Acesso em: ago. 2013.

IMEA - Instituto Mato-Grossense de Economia Agropecuária. Potencial Agropecuário da região Centro Norte de MT, 2013. Disponível em: <http://www.imea.com.br/ upload/pdf/arquivos/Potencial_Produtivo_da_Regiao_Centro_Norte.pdf $>$. Acesso em: jun. 2013.

Kloverpris, J. H. Identification of biomes affected by marginal expansion of agricultural land use induced by increased crop consumption. Journal of Cleaner Production, 17, 463-470, 2009. doi: 10.1016/j.jclepro.2008.08.011

Lopes, C.; Ruchkys, U. Recursos da geodiversidade de São Thomé das Letras - MG e seu uso para mineração e geoconservação: perspectivas para a reconversão desse território mineiro. Desenvolvimento e Meio Ambiente, 35, 335-347, 2015. doi: 10.5380/dma.v35i0.41093

Loureiro, E. M.; Silva, C. J.; Galbiati, C.; Ferreira, J. O.; Nolan, K. S. S. Produção de própolis de Apis mellifera em apiários comerciais de dois municípios de Mato Grosso na Amazônia Meridional. Enciclopédia Biosfera, 9, 28532863, 2013. Disponível em: <http://www.conhecer.org. br/enciclop/2013b/CIENCIAS\%20BIOLOGICAS/Producao $\% 20 \mathrm{de} \% 20$ propolis.pdf $>$.

Marochi M. R.; Oliveira Filho P. C.; Gomes G. S.; Machado A. L. F. Efeitos do uso da terra sobre a produção apícola com destaque para estágios distintos de Floresta Ombrófila Mista. Floresta e Ambiente, 20, 45-54, 2013. doi: 10.4322/ floram.2012.074
Mato Grosso - Secretaria de Estado de Planejamento e Coordenação Geral. Plano de Longo Prazo de Mato Grosso: macro-objetivos, metas globais, eixos estratégicos e linhas estruturantes. In: Prado, J. G. B.; Bertchieli, R.; Oliveira, L. G. (Orgs.). Plano de longo prazo de Mato Grosso. Cuiabá/ MT: Central de Texto, 2012. 108p. Disponível em: <http:// www.seplan.mt.gov.br/mt20/mt20.htm>.

Mato Grosso - Secretaria de Estado de Planejamento e Coordenação Geral - SEPLAN, 2013a. Disponível em: $<$ http://www.seplan.mt.gov.br>. Acesso em: ago. 2013.

Mato Grosso - Secretaria de Estado do Meio Ambiente de Mato Grosso. Desmatamento Dados Municipais, 2013b. Disponível em: <http://www.sema.mt.gov.br/index. php?option $=$ com_content $\&$ view $=$ article $\&$ id $=87 \&$ Item $\mathrm{id}=14>$. Acesso em: ago. 2013.

MDA - Ministério do Desenvolvimento Agrário. 2013. Disponível em: $<$ http://www.mda.gov.br $>$. Acesso em: ago. 2013.

Mendonça, K.; Marchini, L. C.; Souza, B. de A.; Almeida-Anacleto, D. de; Moreti, A. C. de C. C. Caracterização físico-química de amostras de méis produzidas por Apis mellifera $\mathrm{L}$. em fragmento de cerrado no município de Itirapina, São Paulo. Ciência Rural, 38(6), 1748-1753, 2008. doi: 10.1590/S0103-84782008000600040

Mittermeier, R. A.; Mittermeier, C. G.; Brooks, T. M.; Pilgrim, J. D.; Konstant, W. R.; da Fonseca, G. A. B.; Kormos, C. Wilderness and biodiversity conservation. Proceedings of the National Academy of Sciences, 100, 10309-10313, 2003. doi: 10.1073/pnas. 1732458100

Muller, A. C. de P.; Bessa Junior, O. Variação temporal e espacial da cobertura vegetal da floresta ombrófila densa na Área de Proteção Ambiental de Guaraqueçaba no Estado do Paraná, Brasil. Desenvolvimento e Meio Ambiente, 17, 111-119, 2008. doi: 10.5380/dma.v17i0.13415

Pott, A.; Pott V. J. Inventário da flora apícola do Pantanal em Mato Grosso do Sul. Corumbá: Embrapa Pantanal, 1986.

Reis, V. D. A.; Comastri Filho, J. A. Importância da apicultura no Pantanal Sul Mato-Grossense. Documentos 56. Corumbá: Embrapa Pantanal, 1-23, 2003. Disponível em: $<$ http://www.cpap.embrapa.br/publicacoes/online/DOC56. pdf $>$.

Rivero, S.; Almeida, O.; Ávila, S.; Oliveira, W. Pecuária e desmatamento: uma análise das principais causas diretas do 
desmatamento na Amazônia. Nova Economia, 19, 41-66, 2009. doi: 10.1590/S0103-63512009000100003

Sande, S. O.; Crewe, R. M.; Raina, S. K.; Nicolson, S. W.; Gordon, I. Proximity to a forest leads to higher honey yield: Another reason to conserve. Biological Conservation, 142, 2703-2709, 2009. doi: 10.1016/j.biocon.2009.06.023

Santos, C. S.; Ribeiro, A. S. Apicultura uma alternativa na busca do desenvolvimento sustentável. Revista Verde de Agroecologia e Desenvolvimento Sustentável, 4, 1-6, 2006. Disponível em: <http://www.gvaa.com.br/revista/index. php/RVADS/article/view/184/184>.

SEBRAE - Serviço Brasileiro de Apoio às Micro e Pequenas Empresas. Projeto Apicultura, 2013. Disponível em: <http:// www.sebrae.com.br>. Acesso em: 18/08/2013.

Silva, E. R. G.; Galbiati, C. Caracterização taxonômica da flora apícola da região de Cáceres, MT. In: Santos, J. E. dos; Galbiati, C.; Moschini, L. E. (Orgs.). Gestão e Educação Ambiental - Água, Biodiversidade e Cultura. v. 2. São Carlos: Editora RiMa, 2010. p. 186-208.

Silva, L. L.; Costa, R. F.; Campos, J. H. B. C.; Dantas, R. $\mathrm{T}$. Influência das precipitações na produtividade agrícola no Estado da Paraíba. Revista Brasileira de Engenharia Agrícola e Ambiental, 13, 454-461, 2009. Disponível em: $<$ http://www.gvaa.com.br/revista/index.php/RVADS/article/view/184/184>.
Silva-Luz, C. L.; Pirani, J. R. Anacardiaceae. In: Lista de Espécies da Flora do Brasil. Jardim Botânico do Rio de Janeiro, 2015. Disponível em: <http://floradobrasil.jbrj.gov. br/jabot/floradobrasil/FB4382). Acesso em: 20/10/2016.

Simeão, C. M. G.; Silveira, F. A.; Sampaio, I. B. M.; Bastos, E. M. A. F. Pollen analysis of honey and pollen collected by Apis mellifera Linnaeus, 1758 (Hymenoptera, Apidae), in a mixed environment of Eucalyptus plantation and native Cerrado in Southeastern Brazil. Brazilian Journal Biology, 75(4), 821-829. 2015. doi: 10.1590/1519-6984.23513

Sodré, G. S.; Marchini, L. C.; Moreti, A. C. C. C.; Carvalho, C. A. L. Tipos polínicos encontrados em amostras de méis de Apis mellifera em Picos, Estado do Piauí. Ciência Rural, 38, 839-842, 2008. doi: 10.1590/S010384782008000300043

Souza, S. R.; Maciel, M. N. M.; Oliveira, F. A.; Jesuíno, S. A. Caracterização do conflito de uso e ocupação do solo nas áreas de preservação permanente do rio Apeú, nordeste do Pará. Floresta, 42, 701-710, 2012. doi: 10.5380/ rf.v42i4. 19508

Zappi, D. C.; Sasaki, D.; Milliken, W.; Iva, J.; Henicka, G. S.; Biggs, N.; Frisby, S. Plantas vasculares da região do Parque Estadual Cristalino, norte de Mato Grosso, Brasil. Acta Amazonica, 41, 29-38, 2011. doi: 10.1590/S004459672011000100004 